\title{
Relación de las revistas con la investigación: Una amistad de muchos años
}

Saray Córdoba González

Universidad de Costa Rica

Desde que nació la primera revista hace 350 años, esta fue concebida como un medio de comunicación entre los grupos científicos. Primero apareció Le Journal des Sçavans en Francia, en enero de 1665 y ha sido considerado como el precursor de las revistas de humanidades. Posteriormente, en marzo 1665 apareció Philosophical Transactions of the Royal Society of London, solo tres años después del nacimiento de esta sociedad, con la intención de facilitar la comunicación entre sus miembros (Meadows, 1999). Lo más sorprendente es que, desde entonces, la revista científica mantiene esencialmente la misma forma y función.

La Royal Society consideró que era muy importante tener un medio de comunicación que evitara los constantes viajes que hacían sus miembros para obtener datos e información de investigaciones que se encontraban fuera de Inglaterra. Así, fue Henry Oldenburg, secretario de la Royal Society, quien se encargó de recoger las cartas que llegaban a la sociedad y que contenían importantes informes sobre lo que estaban investigando en otros países y sus resultados para publicarlas en la revista. Estas revistas se mantienen vigentes ${ }^{1}$, a pesar de los cambios que la tecnología ha realizado en ellas.

Posteriormente, aparecieron en 1788 The Asiatick Researchers o Transactions of the Society Instituted of Bengal, for Inquiring into the History and Antiquities, the Arts, Sciences and Literature of Asia, como la primera revista asiática, publicada por la Asiatic Society y The American Journal of Science que apareció en Estados Unidos en 1818. Ambas también se siguen publicando. Obsérvese que, en todos estos casos, las publicaciones eran auspiciadas por las sociedades científicas (o Scientific Societies) que trabajaban a través de personas voluntarias y sin fines de lucro, con el fin de crear y diseminar el conocimiento científico (Unesco, 2015, p. 7).

La revista nació en el momento de la Revolución Científica, del Renacimiento y de la Iluminación. Era la época de Galileo, Descartes, Newton, Huygens, Harvey y muchos otras personas que transformaron la ciencia con importantes descubrimientos. La revista nace en ese caldo de cultivo, "de la evolución de un sistema privado de comunicación entre investigadores y de las actas o memorias científicas resultantes de los descubrimientos relatados durante las reuniones y posteriormente impresos para uso de los participantes de los grupos" (Carvalho, 2014, p. 25). De esta manera, la historia nos ofrece la respuesta más clara a la pregunta: ¿es la revista un medio de comunicación para la investigación? Por supuesto que sí, pero podemos profundizar revisando otros datos.

A pesar de que no existe certeza de cuándo empezó la investigación, es en ese momento de renacimiento en que la humanidad se permite invertir esfuerzos para generar conocimiento y que, conjuntamente, se decide crear un medio para comunicarse entre los grupos científicos

1 Le Journal des Sçavans está disponible en: http://www.aibl.fr/publications/periodiques/journal-des-savants/?lang=fr y The Philosophical Transactions of the Royal Society en:

A - http://rsta.royalsocietypublishing.org/content/373/2052?current-issue $=\mathrm{y}$

B - $\underline{\text { http://rstb.royalsocietypublishing.org/content/370/1678 }}$ 
más ágilmente. Esto luego dio como resultado una acelerada reproducción del conocimiento, la proliferación de las academias científicas y el fortalecimiento de las universidades, principalmente en Europa.

Posteriormente, en el siglo XIX, las revistas científicas buscan organizarse más eficientemente con la creación de índices y bibliografías para facilitar el acceso a los artículos que contenían. También, estas adquirieron una periodicidad más regular y se volvieron garantes de la producción científica. Esta es parte del entramado de la comunicación científica que se concibe como la transmisión de conocimiento (Hahn, 2008) por diversos medios, entre ellos el más tradicional y sobresaliente es la revista científica y académica. Tal como lo plantean Harter y Kim (1997), "la ciencia tal como la conocemos, es inimaginable sin la revista académica".

La revista académica y científica es el eslabón final del proceso de generación de conocimiento; sin su aporte, los resultados científicos no se conocerían (Román, 2001). A pesar de que existen otras revistas académicas -como las de divulgación científica y cultural o las técnico-profesionales, tal como las define Latindex y otros medios que han aparecido gracias a internet, como las redes académicas, los blogs, las formas multimedia-, la revista académica y científica es la que prevalece como un medio reconocido y válido en las universidades y en las sociedades científicas de Europa, Norteamérica y América del Sur. Su papel esencial es ser el medio de comunicación de las ciencias; pero, además, es la forma en que se fija el conocimiento y, por tanto, es la vía más importante para evaluar la calidad de la producción científica. Por ello son concebidas como un paso importante en el sistema de evaluación de la ciencia.

No obstante que existen otras formas de comunicación científica, la revista académica y científica se ha consolidado como parte intrínseca de las universidades, sobre todo en América Latina, donde se publican la gran mayoría de las revistas académicas y científicas (Cetto, Alonso, Packer y Aguado, 2015, p. 21). Esto es explicable, porque es allí donde se realiza gran parte de la investigación latinoamericana, generalmente con fondos públicos.

Otra función importante de la revista académica y científica es reforzar la garantía de la propiedad intelectual, porque en la publicación queda en evidencia el autor o autora del descubrimiento (Albornoz, 2006), aun cuando este mismo ya haya sido registrado. Para esto, la publicación que se incluye en la revista ha pasado por la evaluación rigurosa de pares que avalan la calidad, validez y difusión de su contenido. Pero, además, una vez que se ha publicado el artículo en una revista académica, se abre un diálogo que permite que el descubrimiento o aporte original sea legitimado, refutado, negociado o retractado, de manera que el conocimiento sea construido o reconstruido hasta que se logre el consenso de la comunidad científica. Esto permite, a la vez, que ese producto sea utilizado en investigaciones futuras y, en la medida en que se divulgue y se abra su acceso, se reproducirá para hacer crecer el conocimiento nuevo que se genera. Tal como lo califica Albornoz (2006), la revista es una fuente de aprendizaje recíproco.

Por estas razones, todo el equipo editorial de una revista, que va desde el autor o autora hasta el personal editor, pasando por quienes revisan o arbitran y el consejo editorial son investigadores e investigadoras que conocen el campo de especialidad, dominan los procedimientos y métodos de ese campo y el estado de la disciplina en el mundo. Quienes dirigen el proceso de creación y sostenibilidad de una revista deberán comprender, con exactitud, cómo se da el proceso de generación de conocimiento, en sus diferentes estadios y métodos. 
Carvalho (2011) precisa esta relación cuando indica que "la investigación y la revista científica reafirman sus funciones de producir y divulgar el conocimiento con la misión de dar visibilidad a la ciencia y a través del lenguaje cumplir los objetivos, preservando el uso de la escritura y la lectura en diferentes soportes" (p. 39). Que esa amalgama haya subsistido por trescientos cincuenta años resulta muy significativo y, más aún cuando se observa que, a pesar de que aparecen nuevos medios, nuevas versiones y nuevas tecnologías, la revista académica y científica prevalece con los mismos propósitos que la vieron nacer.

\section{Referencias}

Albornoz, M. (2006). Estrategias de comunicación para las revistas científicas. En Diálogo entre editores científicos iberoamericanos: Textos presentados en el primer encuentro iberoamericano de escritores científicos (pp. 11-19). Buenos Aires: CAICyT, CONICET. Parte del libro se encuentra recuperado de http://dialnet.unirioja.es/servlet/ $\underline{\text { libro? } \text { codig }_{0}=438851}$

Carvalho, K. de (2011). Revista científica e pesquisa: Perspectiva histórica. En Revistas científicas dos procesos tradicionais às perspectivas alternativas de comunicação (pp. 23-52). Cotia, SP: Atelié Editorial.

Cetto, A., Alonso-Gamboa, J. O., Packer, A. y Aguado, E. (2015). Enfoque regional a la comunicación científica: Sistemas de revistas en acceso abierto. En Hecho en Latinoamérica (pp. 19-42). Buenos Aires: CLACSO. Recuperado de http://biblioteca. clacso.edu.ar/clacso/se/20150722110704/HechoEnLatinoamerica.pdf

Latindex. (s. f.). Glosario Latindex. Recuperado de http://www.latindex.org/documentos/ Glosario Latindex esp.pdf

Hahn, K. (2008). Talk About Talking About New Models of Scholarly Communication. Journal of Electronic Publishing, 11(1). doi: http://dx.doi.org/10.3998/3336451.0011.108

Harter, S. and Kim, H. (1997). ARCHIVE: Electronic Journals and Scholarly Communication: A Citation and Reference Study. Journal of Electronic Publishing, 3(2). doi: http://dx.doi. org $/ 10.3998 / 3336451.0003 .212$

Meadows, A. J. (1999). A Comunicação científica. Brasília, D.F.: Briquer de Lemos.

Román, A. (2001). La Edición de revistas científicas: Guía de buenos usos. Madrid: CINDOC, CSIC.

Unesco (2015). Scholarly Communication (Module 1). Recuperado de http://unesdoc.unesco. org/images/0023/002319/231938e.pdf 